\title{
Towards a Comprehensive Simulation Model of Malaria Epidemiology and Control
}

T. Smith ${ }^{1}$, N. Maire, A. Ross, M. Penny, N. Chitnis, A. Schapira, A. Studer, B. Genton C.

Lengeler, F. Tediosi, D. de Savigny, M. Tanner

Swiss Tropical Institute

Socinstrasse 57

P.O . Box

CH-4002 Basel

Switzerland

${ }^{1}$ Corresponding author:

email Thomas-A.Smith@unibas.ch 


\section{SUMMARY}

Planning of the control of Plasmodium falciparum malaria leads to a need for models of malaria epidemiology that provide realistic quantitative prediction of likely epidemiological outcomes of a wide range of control strategies. Predictions of the effects of control often ignore medium- and long-term dynamics. The complexities of the Plasmodium life-cycle, and of within-host dynamics, limit the applicability of conventional deterministic malaria models.

We use individual-based stochastic simulations of malaria epidemiology to predict the impacts of interventions on infection, morbidity, mortality, health services use and costs. Individual infections are simulated by stochastic series of parasite densities, and naturally acquired immunity acts by reducing densities. Morbidity and mortality risks, and infectiousness to vectors, depend on parasite densities.

The simulated infections are nested within simulations of individuals in human populations, and linked to models of interventions and health systems. We use numerous field datasets to optimise parameter estimates. By using a volunteer computing system we obtain the enormous computational power required for model fitting, sensitivity analysis, and exploration of many different intervention strategies. The project thus provides a general platform for comparing, fitting, and evaluating different model structures, and for quantitative prediction of effects of different interventions and integrated control programs. 


\title{
INTRODUCTION
}

\begin{abstract}
"Hubris seems to be the chief vice of the model builder without, and even with, field experience. It induces justified scepticism and is counter-productive. It serves only to convince the determinedly non-numerate public health worker that models are a seductive alternative to understanding"(Bradley 1982)

"... and perhaps none will fully understand the behaviour of the whole system as it operates within the economic, social and political constraints of a given society. This may explain some of the difficulties of predicting with sufficient accuracy the likely consequences of any chosen strategy of intervention. And if one cannot predict the consequences of a given strategy, one had no rational basis for choosing between the available alternatives.”(Bailey 1982)
\end{abstract}

Mathematical modeling of malaria transmission dynamics goes back to Ross, who described the population dynamics of malaria and identified entomological thresholds for elimination (Ross 1911). In the 1950s, as the emergence of indoor residual spraying made the elimination of malaria over wide areas conceivable, Ross' work was further developed by Macdonald and others, who provided the mathematical explanation of the effect of reduction of mosquito longevity on malaria transmission, especially through the concept of basic reproductive number(Macdonald 1957). Later models incorporated superinfection, and immunity(Dietz et al. 1974), linked to a field investigation of the feasibility of malaria elimination in the West African savannah(Molineaux and Gramiccia G. 1980). The focus of malaria modelling up until then (reviewed in detail by Bailey(1982)) was on transmission dynamics, and in particular strategies for eliminating the parasite.

Models of malaria dynamics have continued to be developed (e.g. Aron 1988; Struchiner et al. 1989; McKenzie and Bossert 2005) but a very different kind of modeling became prominent in the last few decades of the $20^{\text {th }}$ century, when control programs, especially in Africa, focused on morbidity and mortality rather than transmission. Elimination had proven unrealistic in tropical Africa and had been given up in most tropical countries in Asia and the Americas following severe setbacks. New interventions considered suitable for long-term control were validated through large-scale field trials. This motivated the development of predictive models that use empirical estimates of the effectiveness of interventions, studies on disease burden, and unit costs to quantitate morbidity and mortality and likely cost-effectiveness of interventions. Examples include the work of Goodman et al. (2000) and Rowe et al. (2007). In such models, epidemiological impact is generally inferred from field trial results, which assess only short-term effects using well-controlled delivery systems, and there is no explicit consideration of the dynamics of transmission and immunity, in particular the loss of population immunity that results from decreased transmission.

With a yearly toll of the order of one million lives, malaria remains the main cause of death in young children in Africa(Roll Back Malaria 2005). The disease has therefore become one of the top priorities on the international health agenda. There are a number of efficacious interventions such as insecticide treated nets (ITNs), indoor residual spraying (IRS) and artemisinin-based combination therapy (ACT) but it is not obvious what will be their impact, taking into consideration effects on transmission and health 
system constraints, and so it is not obvious when and how best to deploy and combine them. The resource allocation issues are considerable, with current estimates of US\$ 3.8 to 4.5 billion per year for the total costs of controlling malaria worldwide in accordance with internationally agreed targets (Kiszewski et al. 2007) and the actual annual international transfers for malaria control increasing year by year, reaching USD 600 million in 2006(Roll Back Malaria 2005). Ongoing field research as well as programme monitoring and evaluation is essential to decide on the best strategies in different contexts, but it is not realistic to field-test a large number of combinations of different interventions in different epidemiological and operational settings.

The planning and prioritization of integrated control strategies thus need models that can quantify both short- and long-term effects on transmission, morbidity, mortality, immunity and resistance to medicines and insecticides, at the same time as incorporating field-based data about control, including health system information. Unfortunately there have been very few examples of disease modeling spanning transmission dynamics as well as economic factors, and these have been constrained by the application of limited sets of field data to linear models and limited combinations of interventions(Flessa 2002).

This paper is an overview of the project we are undertaking to develop such comprehensive simulation models of malaria epidemiology and control and to fit them to field data. This project started when we undertook to develop a model to simulate the potential effects of of the introduction of pre-erythrocytic malaria vaccines. The results of this first phase are already published (for an overview see Smith et al. (2006c)). This individual-based stochastic simulation model lends itself to prediction of the potential impact of any malaria intervention strategy under consideration. We are now adapting it for forecasting the epidemiological and economic impact and cost-effectiveness of the whole range of malaria control interventions, singly or combined, applied across a broad range of epidemiological and health system scenarios in malaria endemic areas. In the next section of the paper we outline the major challenges that we face in meeting this ambitious goal. We then describe how we have addressed these challenges to develop a model that can be of real value for malaria control decision-makers and planners.

\section{CHALLENGES}

A general predictive model for malaria epidemiology and control needs a parsimonious structure that mimics malaria biology well enough to capture the mechanisms of action of all the different interventions, and at the same time needs parameter values that reproduce the observed quantative relationships between epidemiological measures. The simultaneous achievement of both these objectives is a major challenge.

The biology and epidemiology of malaria comprises many different dynamic processes with feedback loops. Furthermore, drug treatments, vector control, personal protection and vaccines may all have effects on outcomes that are more complex than the effects on primary infections in the non-immune host. Even if the effect of a vaccine is simply to reduce the force of infection, the short-term consequences in terms of morbidity and mortality are not simply proportional to the reduction in infection rate. There are also 
longer term dynamic effects due to changes in the immune status of the population, benefits due to herd immunity (in the case of vaccines) and community effects of vector control. Predictions of long term effectiveness of interventions in programs also need to consider the health system realities that result in sub-optimal access, targeting accuracy, provider compliance, and consumer adherence. Longer term effects also include the ramifications with regard to health service demands.

The core of a model that can capture all of these is the specification of the properties of an infection. An individual malaria infection can last many months, during which densities of both asexual parasites and gametocytes vary irregularly as consequences mainly of the developmental cycle of the parasite, of immunity, and of antigenic variation. Disease and death are associated with high parasite densities, and the most obvious impact of acquired immunity is in reducing parasite densities. The irregularities in parasite densities lend themselves to treatment as statistical fluctuations. Since the course of each infection is different, and the average behaviour is of less importance than the extent of variation, convincing models for individual infections (e.g. those of Molineaux et al. (2001) and Gatton and Cheng (2004)) have adopted stochastic simulation approaches, rather than treating the development of an individual malaria infection as a deterministic process.

A population model for predicting the impact of interventions that modify parasite densities, such as asexual blood stage vaccination, or treatment with incompletely effective drugs, must explicitly capture relationships with parasite densities, and thus needs to nest within it such a stochastic model for individual infections. This constrains the whole modeling process into an individual-based stochastic framework, which has a number of additional advantages: it is relatively easy to extend individual based simulations indefintely to model very complex systems; stochastic approaches lend themselves to modeling rare events; and stochastic models can be readily extended to model heterogeneities as functions of distributions. However the stochastic approach also leads to difficulties: stochastic models are computationally demanding, and are more difficult to fit to data than deterministic equivalents. The fitting of complex stochastic model to a wide range of different epidemiological outcomes is a challenge, pushing limits both in algorithms of multi-objective optimization and in computational resources.

Fitting of models to data aims to minimize the uncertainty in our predictions, but a further challenge is to identify and quantify the many uncertainties that will remain. Many of these are inherent in any attempt to predict the future. We do not know what new interventions may be discovered and the future costs of malaria control commodities may develop almost unpredictably in reaction to demand, raw material prices, industrial innovation and other factors. Less challenging to quantify are the statistical uncertainties that arise from the difficulty of acquiring enough data. For instance, it is very difficult to quantify natural variation in exposure to mosquito bites, because it is influenced profoundly by a number of factors including for example micro-environment, climate and human behaviour. Other uncertainties, especially those that relate to the human immune response to the parasite, arise because we have not been able to measure the relevant quantities, or do not know what they are. The lack of a proxy measure for protective 
immunity is particularly a problem for vaccine development but it also represents a challenge for the specification of models of disease dynamics. Finally, there are especially large uncertainties with regard to both the performance of health systems and the health seeking behaviours of populations. This is partly because of the limited extent of comprehensive health systems research in malaria endemic areas. Validation of parameters for models of interventions and health systems is constrained to relatively few sites with thorough costing and effectiveness studies of interventions in real life health systems.

\section{EPIDEMIOLOGICAL MODELS}

The first phase of the project (from 2003-2005) focused on developing a single model for malaria epidemiology that reproduces the relevant aspects of malaria biology. We used this to predict the likely impact of pre-erythrocytic vaccines, including projections of the consequences of introducing such vaccines into the Expanded Program on Immunization, for both malaria epidemiology(Maire et al. 2006c) and vaccine costeffectiveness(Tediosi et al. 2006a). This required us to develop simulations of the malaria transmission cycle, of the course of individual infections, of the risk of morbidity and mortality as a function of transmission intensity, and of the impact of the health systems on malaria in endemic settings. The details of the models used, including their justification in terms of biology and epidemiology have been published as a supplement to the American Journal of Tropical Medicine and Hygiene, of which the first paper provides an overview, together with the mathematical description(Smith et al. 2006c).

The simulated time-courses of parasite densities are based on a description of parasite densities in neurosyphilis patients who received therapeutic $P$. falciparum infections. By placing parasite densities as the centre of a causal web linking infection with its downstream effects (Figure 1), we are able to reproduce key features of malaria biology that are difficult to capture with other approaches.

For instance, in our models as in the real world, the main effect of naturally acquired immunity to malaria is to reduce infection density, rather than persistence of the infection(Sama et al. 2004; Sama et al. 2006). High parasite densities are also a trigger of clinical malaria and are quantitatively related to severity of disease, and so in our simulations these events occur when parasite densities exceed given thresholds. Similarly, the probability that a mosquito is infected when feeding on an infected human depends on the gametocyte density.

So far we have concentrated on simulating malaria epidemiology in sites where there are field-based measurements of the seasonal pattern in the entomological inoculation rate (EIR). Treating this average EIR as a known quantity, but allowing for temporal variations and the influence of host age (larger persons receive more bites(Port et al. 1980)), we introduce infections into a population of simulated humans via a stochastic process dependent on the EIR(Smith et al. 2006d) and then sample the subsequent parasite densities using a 5-day time steps. We estimate the sampling distributions for 
the parasite densities from the time-trends in malaria therapy data and from immunity parameters determined from each simulated individual's history of infection(Maire et al. 2006b).

Because the presence of $P$. falciparum in the blood does not prevent a new infection (superinfection), the population simulations need to simulate not just single infections but also recurrent and concurrent multiple infections.

We have treated infection events as a Poisson process, and our requirement that measurements of the EIR should be available, restricted us to simulating only areas of intense endemic transmission. This Poisson assumption neglects the clustering in infection that certainly occurs in malaria. Some people are more attractive to mosquitoes than others(Knols et al. 1995), and some live in microenvironments where they are more exposed. However the approach can readily be extended to include additional heterogeneity in the infection process, for instance by replacing the Poisson process with a negative binomial one. This will be particularly important in simulating low transmission areas and in predicting the outcome of highly effective combinations of interventions, where models of homogeneity are likely to give over-optimistic forecasts of local elimination.

\section{MODELS OF INTERVENTION STRATEGIES}

We plan to define and simulate a minimal essential set of interventions, intervention combinations and scale-up strategies for malaria control. Table 2 lists in outline some among the many possible strategies, each of which will be specified in detail, and for each of which a model for action of the interventions must be incorporated into the simulations. The appropriateness of any intervention strategy will depend on the epidemiological setting and health system.

For case management of malaria we include the effects on morbidity, mortality and transmission. Models of health system effectiveness in intervention delivery are an important part of the simulation of any preventive and curative intervention, since the effectiveness can be affected by constraints both on the provider and the consumer side. We also consider the reduced burden on the health system resulting from preventive interventions and case management. Quantitative modeling of the feedbacks in health systems is in its infancy, and so far we have completed only a basic model based on data from Tanzania(Tediosi et al. 2006b). We are currently revising this to incorporate improved simulations of diagnosis, drug action, of referral, of the contribution of the informal sector, and of the implications of intermittent preventive treatment as well as transmission reducing interventions.

Our analyses of the results of field trials of a pre-erythrocytic vaccine were compatible with a simple model, namely that such vaccines block a percentage of sporozoite inoculations from reaching the blood. This percentage may vary between human hosts, but it seems no-one is completely protected from infection by such a vaccine(Maire et al. 2006a). 
We can model mosquito-stage transmission blocking vaccines similarly by assuming a defined proportionate reduction in the probability that a mosquito becomes infected from any one feed. We have also modelled blood stage vaccines by assuming the immediate effect to be reduction in parasite densities. This assumption may be an oversimplification as vaccine-induced reduction in asexual blood stage challenge very likely has complex effects on parasite dynamics. We are developing improved within-host models based on stochastic simulation to include interaction between asexual parasites and host immune responses. We anticipate that these should provide us with models for asexual blood stage vaccination and for the action of antimalarial drugs that capture these dynamics.

We base our models for the effects of personal protection and vector control on discretetime models of the mosquito feeding cycle(Saul et al. 1990; Killeen and Smith 2007). We have generalized these models to incorporate the heterogeneity of human and nonhuman animal hosts, and explicitly model the different stages of the mosquito feeding cycle that can be targeted by different interventions, allowing the duration of the feeding cycle to vary(Chitnis et al. 2007). The model is being parameterized to simulate the effects of Indoor Residual Spraying (IRS) and for simulating effects of Insecticide Treated Nets (ITNs) at various performance levels of the health system. Combinations of both interventions can also be simulated. For the latter, we are considering the dynamics of mosquito net coverage, to include the effects of deterioration of nets and loss of insecticidal effects. These models will be validated by simulating field trials of ITNs. Once these models in the overall stochastic simulation platform we will be able not only to simulate plausible vector control strategies, but also integrated control involving multiple interventions.

\section{SOFTWARE AND FITTING TO DATA}

Our stochastic simulation models are highly computation-intensive, and are implemented in the general purpose programming languages Fortran 95 and $\mathrm{C}++$ for performance and flexibility. The specifications of the scenario to be simulated are provided in an input file written in Extensible Markup Language (XML), and output is returned in the form of text files containing predictions of age- and time- specific epidemiological quantities (infection prevalence, density, multiplicity of infection, incidence and severity of morbidity, mortality, and intervention coverages).

In our initial overview publication we listed a total of 38 parameters in the overall model of malaria epidemiology. Considering that this comprises simulations of the whole transmission cycle, relevant aspects of immunity, and of a wide range of morbidity and mortality outcomes, this is not excessive, but optimisation within a space of this dimension is challenging.

Some of the current components of this model are estimated independently of the others. Six of the parameters are part of a statistical model for how anaemia depends on infection status(Carneiro et al. 2006). This anaemia component does not feed back into the rest of the model. Nor do values of five parameters that quantify infectivity of human hosts to mosquitoes affect the estimates of the rest of the parameters and these were estimated in stand-alone exercises (Ross et al. 2006a; Killeen et al. 2006). Two other components of 
the models are estimated directly from field data: the time-course of parasite densities in individual infections in a previously untreated host is based on a statistical description of malaria therapy profiles(Maire et al. 2006b); and the relationship between the force of infection (parasitological inoculation rate) and the EIR is estimated from the data of a field study that specifically addressed this question(Beier et al. 1994). Malaria attributable neonatal mortality rates are taken from systematic reviews (Ross and Smith 2006).

The other components of the model, corresponding to 25 different unknown quantities (parameters), are fitted by treating the stochastic simulation as an implicit statistical model (a list of these parameters is given by Smith et al (2006c)). A total of 61 standard scenarios have been constructed, corresponding to sites where both the pattern of transmission and one or more epidemiological variables have been measured (Table 1). These epidemiological variables define a set of 10 different objective functions (likelihoods or sums of squares) corresponding to different epidemiological outcome measures that must be optimised. In the first phase of the project we were able to fit our model to all of these objectives using a simulated annealing algorithm. We used a desktop-grid approach to distribute the simulations across our local computer network, using up to 40 processors at any one time. However this was only possible by fitting the different objectives and corresponding model components sequentially, each conditional on the upstream objectives. Even then, many of the optimisation runs required several weeks of computing. The computational demands were particularly high for the large number of scenarios used for predicting rates of severe disease and mortality. Since these are relatively rare events, populations of many tens of thousands of individuals are needed to provide stable predictions of rates that can be compared with field data.

In the current phase of the project the computational demands are even higher because we are developing and comparing a number of alternative model formulations. These involve explicit simulations of longitudinal patterns of parasitemia within hosts; effects of heterogeneity in transmission (to simulate areas of focal transmission); decay in acquired immunity against malaria; parasite drug resistance; and different models for preventive and curative interventions, singly and combined.

To optimise all these models we are harnessing the spare capacity of computers made available across the internet by volunteers via the Berkeley Open Infrastructure for Network Computing (BOINC) (http://boinc.berkeley.edu/). The BOINC middleware manages the allocation of tasks to volunteer computers (scheduling), information exchange about the project, and the corresponding security issues both for our servers and for the volunteers. Using this infrastructure we can fit many models in parallel using genetic algorithms(Holland 1975). Each iteration of the fitting process involves sending each of the 61 standard scenarios to the volunteers, combining their results to give loss functions for each of the 10 objectives, and computing an overall loss function. The project server generates new parameterisations and tracks convergence (Figure 2). Within this framework, many different optimizations can be carried out in parallel, with the main limitations being the server capacity, the minimum turnaround time on the work units sent out to the volunteers, and the capacity of the research team to process the 
results. These in turn mean the time needed to perform one optimization remains several weeks or months with the current algorithm.

\section{OUTLOOK}

The development of a comprehensive simulation model for malaria epidemiology and control requires a substantial trans-disciplinary effort, and extraordinary computing power. To this end, the Swiss Tropical Institute has assembled a research team that draws on skills from epidemiology, clinical medicine, immunology, entomology, laboratory sciences, health systems, , mathematics, statistics, economics, and computer sciences. The project is guided by an international technical advisory group of malaria and modeling experts. Finally, development of a valid and useful comprehensive model such as this requires access to numerous large and diverse field data sets informing the many dimensions required to validate the model. For this we are fortunate to have the collaboration of many partners and field projects from areas with endemic malaria and intend to further broaden this to obtain the strongest possible validation.

It seems unlikely that any of the intervention strategies we are simulating will prove to be a magic bullet that will solve the problem of malaria for good. On the other hand, the application of all existing control interventions in all endemic areas is likely to be highly cost-ineffective. Practical malaria control experience indicates that the application of a well selected limited menu of interventions in a given area can capitalise on the advantages of each one. Modeling integrated control strategies will thus be of great utility for informing malaria control programmes about which strategies are most likely to be cost-effective under specific conditions.

In the light of the many uncertainties inherent in such an exercise, it is crucial to supplement the model predictions with sensitivity analyses, development of new models, and validation of model outputs against accumulated field data. The availability of the www.malariacontrol.net volunteer computing resources enables us not only to fit many models, but also to carry out the extensive analysis of model predictions, and in the same way as climate modelers now make predictions from ensembles of different models, we will be most confident about the predictions of disease models if we see that they are consistent across a range of different sets of assumptions. To judge how robust are the simulation results we can also perform probabilistic sensitivity analysis (PSA) to identify the sensitivity of model predictions to the different parameters, thus helping to identify which model assumptions are most important in determining the predictions, and where further data may be needed. By including costing parameters in the PSA we can also analyse acceptability curves for specific intervention strategies.

The final set of model results will be made publicly accessible and will include userinterfaces tailored to a various categories of users including health planners, control program managers international policy makers, researchers and industry, who will be able to query specific eco-epidemiologic and health system scenarios. This will enable models to take their place as important tools for rational planning of malaria control. 


\section{ACKNOWLEDGEMENTS}

This project depends on the efforts of many researchers and field staff engaged in malariological fieldwork in endemic areas, and many thousands of volunteers who make their computers available to malariacontrol.net.

The first phase of the study was funded by the PATH Malaria Vaccine Initiative and GlaxoSmithKline Biologicals S.A. The second phase is supported by the Bill \& Melinda Gates Foundation. This publication and the contents hereof do not necessarily reflect the endorsement, opinion, or viewpoints of the PATH Malaria Vaccine Initiative, GlaxoSmithKline Biologicals S.A., or the Bill \& Melinda Gates Foundation. 
Table 1. Summary of datasets used for parameterising the epidemiological models

\begin{tabular}{|c|c|c|c|c|c|}
\hline Objective & $\begin{array}{l}\text { Inclu- } \\
\operatorname{ded}^{\&}\end{array}$ & $\begin{array}{l}\text { Sources of } \\
\text { data }\end{array}$ & $\begin{array}{l}\text { Number } \\
\text { of scen- } \\
\text { arios }^{\$}\end{array}$ & $\begin{array}{l}\text { Number of } \\
\text { data } \\
\text { points* }\end{array}$ & $\begin{array}{l}\text { Ref- } \\
\text { erence } \\
\text { for the } \\
\text { analysis }\end{array}$ \\
\hline $\begin{array}{l}\text { Parasite densities in primary } \\
\text { infections by age of infection }\end{array}$ & No & $\begin{array}{l}\text { (Collins } \\
\text { and Jeffery } \\
\text { 1999) }\end{array}$ & n.a. & 47 patients & $\begin{array}{l}\text { (Maire } \\
\text { et al. } \\
\text { 2006b) }\end{array}$ \\
\hline $\begin{array}{l}\text { Infectivity of humans to } \\
\text { mosquitoes by history of } \\
\text { parasite density }\end{array}$ & No & $\begin{array}{l}\text { (Collins } \\
\text { and Jeffery } \\
\text { 2003) }\end{array}$ & n.a. & $\begin{array}{l}730 \text { feeding } \\
\text { experiments }\end{array}$ & $\begin{array}{l}\text { (Ross et } \\
\text { al. } \\
\text { 2006a) }\end{array}$ \\
\hline $\begin{array}{l}\text { Incidence of new infection in } \\
\text { previously treated children\# }\end{array}$ & No & $\begin{array}{l}\text { (Beier et } \\
\text { al. 1994) }\end{array}$ & 21 & 62 & $\begin{array}{l}\text { (Smith } \\
\text { et al. } \\
\text { 2006a) }\end{array}$ \\
\hline $\begin{array}{l}\text { Age pattern of incidence of } \\
\text { new infection in treated } \\
\text { individuals\# }\end{array}$ & Yes & $\begin{array}{l}\text { (Molineaux } \\
\text { and } \\
\text { Gramiccia } \\
\text { G. 1980) }\end{array}$ & 1 & 12 & $\begin{array}{l}\text { (Smith } \\
\text { et al. } \\
\text { 2006a) }\end{array}$ \\
\hline $\begin{array}{l}\text { Age- and seasonal patterns of } \\
\text { prevalence of infection\# }\end{array}$ & Yes & $\begin{array}{l}\text { (Molineaux } \\
\text { and } \\
\text { Gramiccia } \\
\text { G. 1980) }\end{array}$ & 6 & 563 & $\begin{array}{l}\text { (Maire } \\
\text { et al. } \\
\text { 2006b) }\end{array}$ \\
\hline Age- and seasonal patterns of & Yes & (Molineaux & 6 & 563 & (Maire \\
\hline
\end{tabular}




\begin{tabular}{|c|c|c|c|c|c|}
\hline parasite density\# & & $\begin{array}{l}\text { and } \\
\text { Gramiccia } \\
\text { G. 1980) }\end{array}$ & & & $\begin{array}{l}\text { et al. } \\
\text { 2006b) }\end{array}$ \\
\hline $\begin{array}{l}\text { Age pattern of number of } \\
\text { concurrent infections\# }\end{array}$ & Yes & $\begin{array}{l}\text { (Maire et } \\
\text { al. 2006b; } \\
\text { Owusu- } \\
\text { Agyei et } \\
\text { al. 2002) }\end{array}$ & 1 & 12 & $\begin{array}{l}\text { (Maire } \\
\text { et al. } \\
\text { 2006b) }\end{array}$ \\
\hline $\begin{array}{l}\text { Age pattern of incidence of } \\
\text { clinical malaria\# }\end{array}$ & Yes & $\begin{array}{l}\text { (Trape and } \\
\text { Rogier } \\
\text { 1996; } \\
\text { Kitua et al. } \\
\text { 1996) }\end{array}$ & 3 & 31 & $\begin{array}{l}\text { (Smith } \\
\text { et al. } \\
\text { 2006b) }\end{array}$ \\
\hline $\begin{array}{l}\text { Age pattern of threshold } \\
\text { parasite density for clinical } \\
\text { attacks\# }\end{array}$ & Yes & $\begin{array}{l}\text { (Rogier et } \\
\text { al. 1996) }\end{array}$ & 1 & 13 & $\begin{array}{l}\text { (Smith } \\
\text { et al. } \\
\text { 2006b) }\end{array}$ \\
\hline $\begin{array}{l}\text { Hospitalisation rate in relation } \\
\text { to prevalence in children }\end{array}$ & Yes & $\begin{array}{l}\text { See (Ross } \\
\text { et al. } \\
\text { 2006b) }\end{array}$ & 26 & $10^{\mathrm{E}}$ & $\begin{array}{l}\text { (Ross et } \\
\text { al. } \\
\text { 2006b) }\end{array}$ \\
\hline Age pattern of hospitalisation\# & Yes & $\begin{array}{l}\text { (Marsh and } \\
\text { Snow } \\
\text { 1999) }\end{array}$ & 4 & 12 & $\begin{array}{l}\text { (Ross et } \\
\text { al. } \\
\text { 2006b) }\end{array}$ \\
\hline Malaria specific mortality in & Yes & (Snow et & 9 & 9 & (Ross et \\
\hline
\end{tabular}




\begin{tabular}{|l|l|l|l|l|l|}
\hline children\# & & al. 1997) & & & al. \\
& & & & & 2006b) \\
\hline Infant mortality rate\# & Yes & See (Ross & 11 & 11 & (Ross et \\
& & $\begin{array}{l}\text { et al. } \\
\text { 2006b). }\end{array}$ & & & al. \\
& & & & $2006 \mathrm{~b})$ \\
\hline
\end{tabular}

\& This column indicates whether this objective is included in the simultaneous multiobjective fitting of the model, or whether the data are used to seperately parameterise model components.

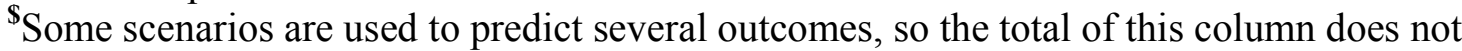
equal the total of 61 scenarios involved in fitting the models.

*The number of data points is the sum over all scenarios and simulated survey periods of the number of age groups into which the data were disaggregated for comparison with the model predictions.

"In relation to the seasonal pattern in the EIR

${ }^{\mathfrak{E}}$ Model predictions for this objective are compared with interpolations between the field data points.

n.a. not applicable 
Table 2. Examples of interventions to combine in integrated control programs

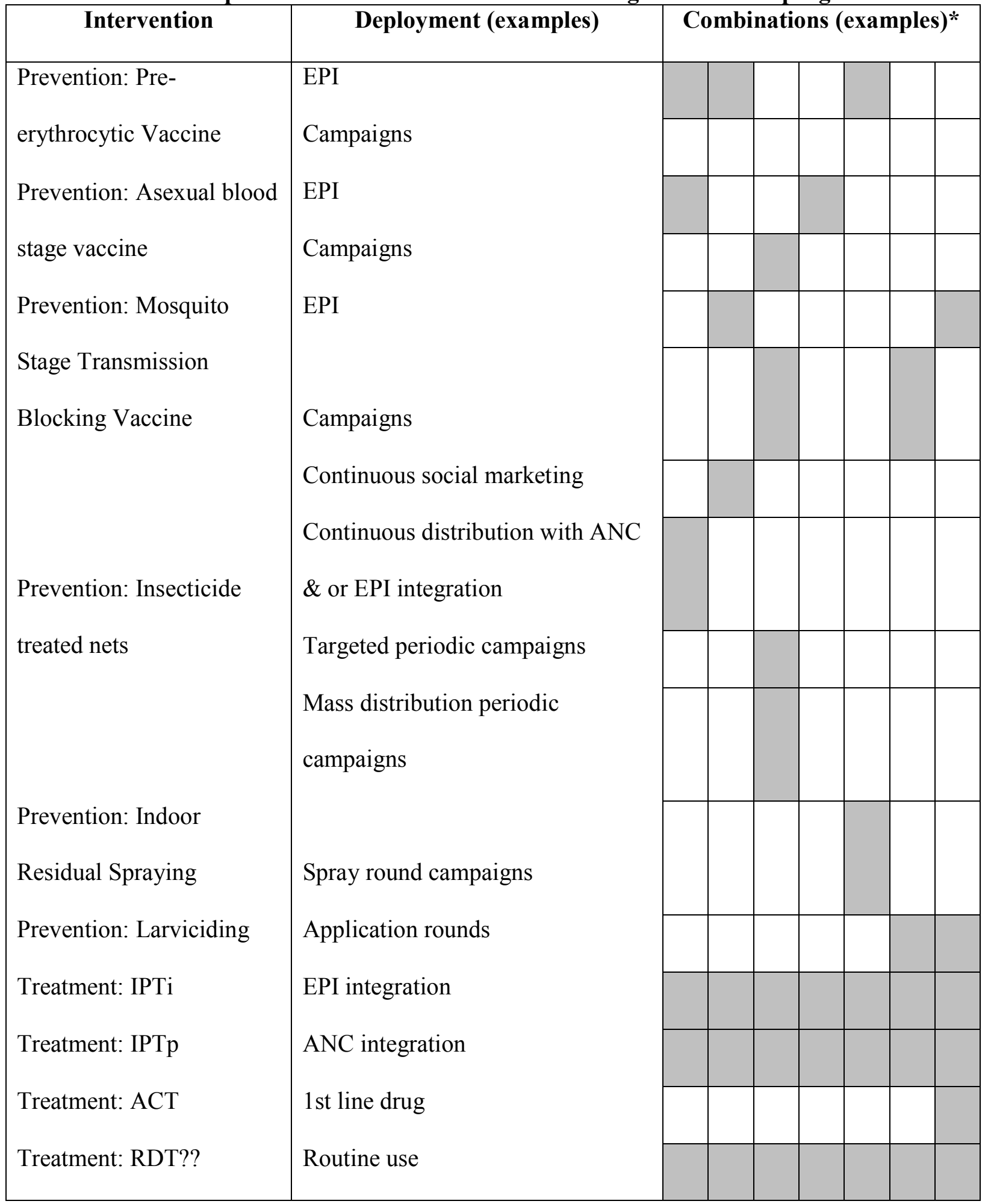

*each column corresponds to one strategy of integrated or combined intervention programs, making use of each of the interventions indicated by shading. The project will 
simulate a very wide range of such combinations but not all are possible: we will concentrate on analysing those most likely to be adapted to the particular epidemiological setting.

Abbreviations: IPTi: intermittent preventive treatment in infants; IPTp: intermittent preventive treatment in pregnancy; ACT: artemisinin combination therapy; RDT: rapid diagnostic test. 


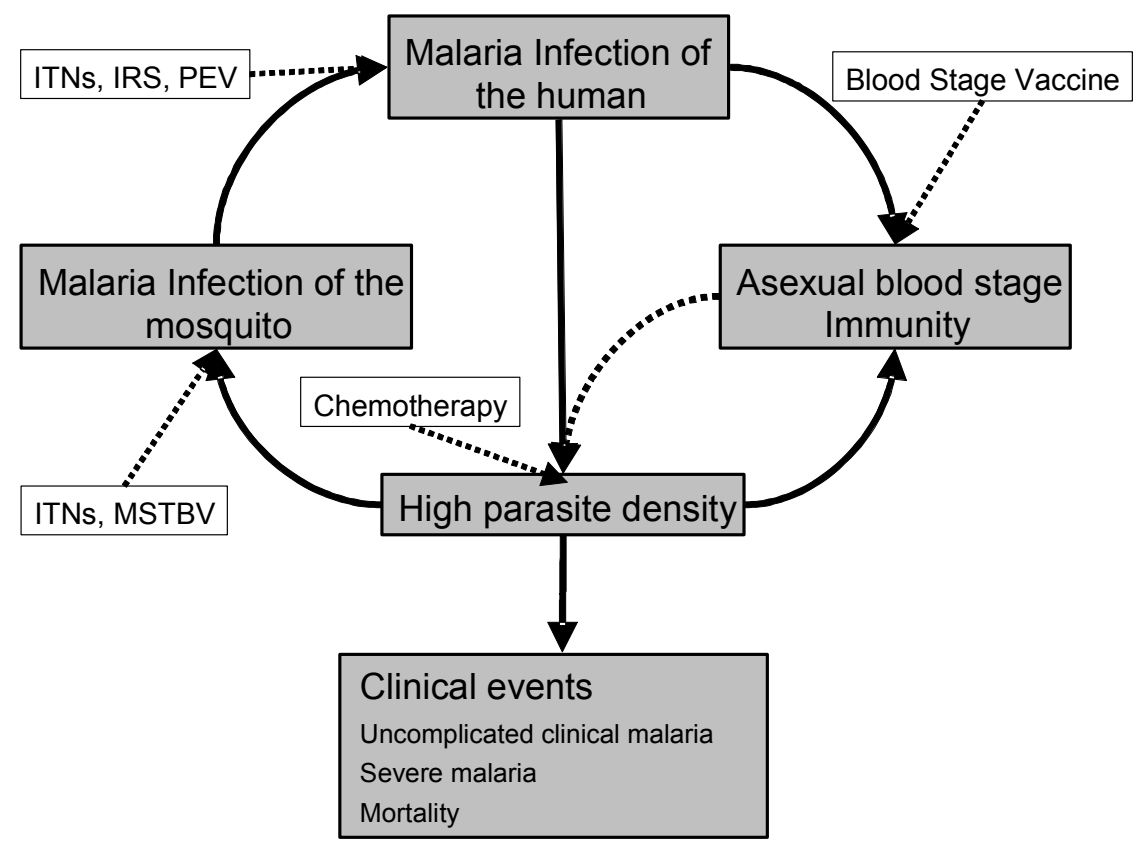

Figure 1. Key causal factors and outcomes in the models of malaria epidemiology and interventions.

Arrows correspond to the main relationships modelled. Dashed arrows correspond to negative relationships. 


\section{Figure 2. Loss function by iteration (example run)}

Each point corresponds to a single value of the weighted average of the loss functions for each of the 61 scenarios for a single model parameterisation. This run illustrates slow convergence when many similar parameterisations are run in parallel using the current genetic algorithm.

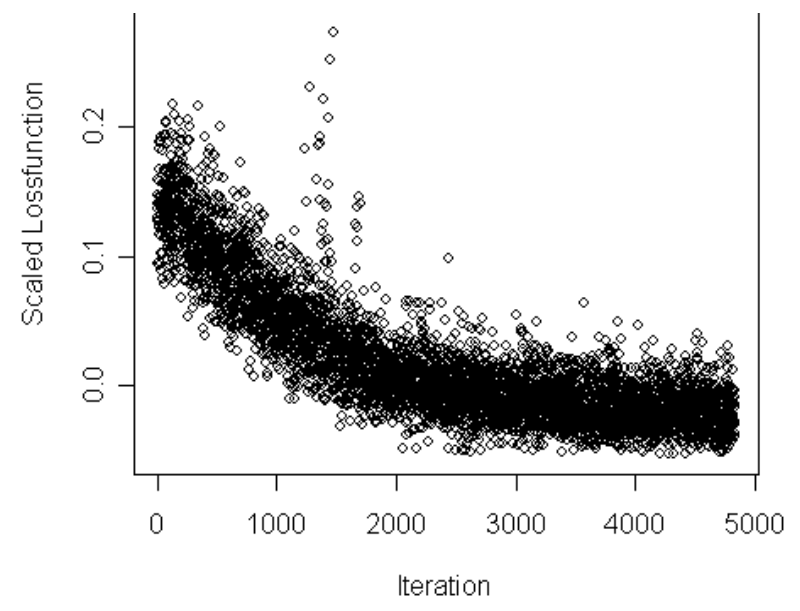




\section{REFERENCE LIST}

Aron JL (1988) Mathematical-Modeling Of Immunity To Malaria. Mathematical Biosciences 90, 385-396.

Bailey N (1982) 'The biomathematics of malaria.' (Charles Griffin and Co Ltd: London)

Beier JC, Oster CN, Onyango FK, Bales JD, Sherwood JA, Perkins PV, Chumo DK, Koech DV, Whitmire RE, Roberts CR (1994) Plasmodium falciparum incidence relative to entomologic inoculation rates at a site proposed for testing malaria vaccines in western Kenya. Am J Trop Med Hyg 50, 529-536.

Bradley DJ (1982) Epidemiological models theory and reality. In 'The population dynamics of infectious diseases: theory and application'. (Ed. RM Anderson) (Chapman Hall; 1982: London and New York)

Carneiro I, Smith T, Lusingu J, Malima R, Utzinger J, Drakeley C (2006) Modeling the relationship between the population prevalence of Plasmodium falciparum malaria and anemia. Am J Trop Med Hyg 75 (Suppl 2), 82-89.

Chitnis N, Steketee RW, Smith T (2007) A Mathematical Model for the Dynamics of Malaria in Mosquitoes Feeding on a Heterogeneous Host Population. Journal of Biological Dynamics.

Collins WE, Jeffery GM (1999) A retrospective examination of sporozoite- and trophozoite-induced infections with Plasmodium falciparum: development of 
parasitologic and clinical immunity during primary infection. Am.J Trop Med Hyg 61, 419.

Collins WE, Jeffery GM (2003) A retrospective examination of mosquito infection on humans infected with Plasmodium falciparum. Am J Trop Med Hyg 68, 366-371.

Dietz K, Molineaux L, Thomas A (1974) A malaria model tested in the African savannah. Bull World Health Org 50, 347-357.

Flessa S (2002) 'Malaria und AIDS: Gesundheitökonomische Analysen auf der Grundlage von Disease Dynamic Modellen.' (Hans Jacobs: Lage)

Gatton ML, Cheng Q (2004) Modeling the development of acquired clinical immunity to Plasmodium falciparum malaria. Infect.Immun. 72, 6538-6545.

Goodman CA, Coleman PG, Mills A (2000) 'Economic analysis of malaria control in sub-Saharan Africa.' (Global Forum for Health Research: Geneva)

Holland J (1975) 'Adaptation In Natural And Artificial Systems.' (University of Michigan Press:

Killeen GF, Ross A, Smith T (2006) Infectiousness of malaria-endemic human populations to vectors. Am J Trop Med Hyg 76 (Suppl 2), 38-45.

Killeen GF, Smith TA (2007) Exploring the contributions of bed nets, cattle, insecticides and excitorepellency to malaria control: a deterministic model of mosquito host-seeking behaviour and mortality. Trans.R.Soc.Trop.Med.Hyg. 101, 867-880. 
Kiszewski A, Johns B, Schapira A, Delacollette C, Crowell V, Tan-Torres T, Amenashewa B, Teklehaimanot A, Nafo-Traoré F (2007) Estimated GlobalResources Needed to Attain International Malaria Control Goals. Bull World Health Organ.

Kitua A, Smith T, Alonso PL, Masanja H, Urassa H, Menendez C, Kimario J, Tanner M (1996) Plasmodium falciparum malaria in the first year of life in an area of intense and perennial transmission. Trop Med Int Health 1, 475-484.

Knols BG, De Jong R, Takken W (1995) Differential attractiveness of isolated humans to mosquitoes in Tanzania. Trans R Soc Trop Med Hyg 89, 604-606.

Macdonald G (1957) 'The epidemiology and control of malaria.' (Oxford University Press: London)

Maire N, Aponte JJ, Ross A, Thompson R, Alonso P, Utzinger J, Tanner M, Smith T (2006a) Modeling a field trial of the RTS,S/AS02A malaria vaccine. Am.J.Trop.Med.Hyg. 75, 104-110.

Maire N, Smith T, Ross A, Owusu-Agyei S, Dietz K, Molineaux L (2006b) A model for natural immunity to asexual blood stages of Plasmodium falciparum malaria in endemic areas. Am.J.Trop.Med.Hyg. 75, 19-31.

Maire N, Tediosi F, Ross A, Smith T (2006c) Predictions of the epidemiologic impact of introducing a pre-erythrocytic vaccine into the expanded program on immunization in sub-Saharan Africa. Am.J.Trop.Med.Hyg. 75, 111-118. 
Marsh K, Snow R (1999) Malaria transmission and morbidity. Parassitologia 41, 241246.

McKenzie FE, Bossert WH (2005) An integrated model of Plasmodium falciparum dynamics. J Theor.Biol. 232, 411-426.

Molineaux L, Diebner HH, Eichner M, Collins WE, Jeffery GM, Dietz K (2001)

Plasmodium falciparum parasitaemia described by a new mathematical model.

Parasitology 122, 379-391.

Molineaux L, Gramiccia G. (1980) 'The Garki Project.' (World Health Organisation: Geneva)

Owusu-Agyei S, Smith T, Beck H-P, Amenga-Etego L, Felger I (2002) Molecular epidemiology of Plasmodium falciparum infections among asymptomatic inhabitants of a holoendemic malarious area in northern Ghana. Trop Med Int Health 7, 421-428.

Port GR, Boreham PFL, Bryan JH (1980) The relationship of host size to feeding by mosquitos of the Anopheles-gambiae giles complex (Diptera, Culicidae). Bull Entomol Res 70, 133-144.

Rogier C, Commenges D, Trape JF (1996) Evidence for an age-dependent pyrogenic threshold of Plasmodium falciparum parasitemia in highly endemic populations. Am J Trop Med Hyg 54, 613-619.

Roll Back Malaria WaU (2005) 'World Malaria Report 2005.' (WHO and UNICEF: Geneva) 
Ross A, Killeen GF, Smith T (2006a) Relationships of host infectivity to mosquitoes and asexual parasite density in Plasmodium falciparum . Am J Trop Med Hyg 75 (Suppl 2), $32-37$.

Ross A, Smith T (2006) The effect of malaria transmission intensity on neonatal mortality in endemic areas. Am J Trop Med Hyg 75 (Suppl 2), 74-81.

Ross A, Maire N, Molineaux L, Smith T (2006b) An epidemiologic model of severe morbidity and mortality caused by Plasmodium falciparum. Am.J.Trop.Med.Hyg. 75, 6373.

Ross R (1911) 'The prevention of malaria.' (Murray: London)

Rowe A, Steketee R, Arnold F, Wardlaw T, Basu S, Bakyaita N, Lama M, Winston C, Lynch M, Cibulskis R, Shibuya K, Ratcliffe A, Nahlen B, for the Roll Back Malaria Monitoring and Evaluation Refernce Group (2007) Evaluating the impact of malaria control efforts on mortality in sub-Saharan Africa. TMIH submitted.

Sama W, Killeen G, Smith T (2004) Estimating the duration of Plasmodium falciparum infection from trials of indoor residual spraying. Am J Trop Med Hyg 70, 625-634.

Sama W, Owusu-Agyei S, Felger I, Dietz K, Smith T (2006) Age and seasonal variation in the transition rates and detectability of Plasmodium falciparum malaria. Parasitology 132, 13-21. 
Saul A, Graves PM, Kay BH (1990) A cyclical feeding model for pathogen transmission and its application to determine vectorial capacity from vector infection-rates. Journal of Applied Ecology 27, 123-133.

Smith T, Maire N, Dietz K, Killeen GF, Vounatsou P, Molineaux L, Tanner M (2006a) Relationships between the entomological inoculation rate and the force of infection for Plasmodium falciparum malaria. Am J Trop Med Hyg 75 (Suppl 2), 11-18.

Smith T, Ross A, Maire N, Rogier C, Trape JF, Molineaux L (2006b) An epidemiological model of the incidence of acute illness in Plasmodium falciparum malaria. Am J Trop Med Hyg 75 (Suppl 2), 56-62.

Smith T, Killeen GF, Maire N, Ross A, Molineaux L, Tediosi F, Hutton G, Utzinger J, Dietz K, Tanner M (2006c) Mathematical modeling of the impact of malaria vaccines on the clinical epidemiology and natural history of Plasmodium falciparum malaria: Overview. Am.J.Trop.Med.Hyg. 75, 1-10.

Smith T, Maire N, Dietz K, Killeen GF, Vounatsou P, Molineaux L, Tanner M (2006d) Relationship between the entomologic inoculation rate and the force of infection for Plasmodium falciparum malaria. Am.J.Trop.Med.Hyg. 75, 11-18.

Snow R, Omumbo J, Lowe B, Molyneux CS, Obiero JO, Palmer A, Weber MW, Pinder M, Nahlen B, Obonyo C, Newbold C, Gupta S, Marsh K (1997) Relation between severe malaria morbidity in children and level of Plasmodium falciparum transmission in Africa [see comments]. Lancet 349, 1650-1654. 
Struchiner CJ, Halloran ME, Spielman A (1989) Modeling malaria vaccines. I: New uses for old ideas. Math.Biosci. 94, 87-113.

Tediosi F, Hutton G, Maire N, Smith TA, Ross A, Tanner M (2006a) Predicting the costeffectiveness of introducing a pre-erythrocytic malaria vaccine into the expanded program on immunization in Tanzania. Am.J.Trop.Med.Hyg. 75, 131-143.

Tediosi F, Maire N, Smith T, Hutton G, Utzinger J, Ross A, Tanner M (2006b) An approach to model the costs and effects of case management of Plasmodium falciparum malaria in sub-saharan Africa. Am.J.Trop.Med.Hyg. 75, 90-103.

Trape JF, Rogier C (1996) Combating malaria morbidity and mortality by reducing transmission. Parasitol Today 12, 236-240. 